\title{
Los medios de comunicación en la construcción de ciudadanía
}

\section{The media in the construction of citizenship}

\author{
MSc, Benjamín Wilson León Valle ${ }^{1}$ \\ Misiowill@yahoo.es \\ MSc, Divar Sebastián Castro Loor \\ d.castro.loor@gmail.com
}

Recibido: 1/04/2018, Aceptado: 1/06/2018

\begin{abstract}
RESUMEN
Es innegable que la ciudadanía se alimenta de información presentada por los medios de comunicación, estos contribuyen a elaborar marcos referenciales que conducen a la población en generar en cierto sentido y un saber respecto a lo que sucede en el quehacer diario. Estos son percibidos como instituciones confiables, refuerzan la credibilidad de la ciudadanía con la información que generan, reproducen y transmiten. El marco constitucional presenta a los medios de comunicación, y a la comunicación; como un servicio que debe articularse transversalmente al proceso de construcción del país incluyente y participativo, sin embargo, en la práctica estos lineamientos, no llegan a todos los estamentos de la sociedad, por la agenda personal o mediática. La metodología implementada es el método descriptivo, los instrumentos de investigación son: entrevista, encuesta y observación; apoyados por la ficha de observación, para realizar una buena descripción del objeto de estudio.
\end{abstract}

Palabras clave: Comunicación, ciudadanía, construcción, Constitución

\section{ABSTRACT}

It is undeniable that citizens are nourished by information presented by the media, they contribute to developing frameworks that lead the population to generate in a certain sense and a knowledge about what happens in the daily work. These are perceived as reliable institutions, reinforce the credibility of the citizenship with the information they generate, reproduce and transmit. The constitutional framework presents the media and communication; as a service that must be articulated transversally to the process of construction of the inclusive and participatory country, however, in practice these guidelines do not reach all levels of society by personal or media agenda. The methodology implemented is the descriptive method, the research instruments are: interview, survey and observation; supported by the observation card, to make a good description of the object of study.

\footnotetext{
1 Universidad Estatal Península de Santa Elena, Ecuador

2 Universidad Estatal Península de Santa Elena, Ecuador
} 
Keywords: Communication, citizenship, construction, Constitution

\section{Introducción}

Los medios de comunicación tienen un rol histórico en la construcción de la sociedad, en lo cotidiano se han convertido en generadores de opinión tanto social como pública, es innegable que la ciudadanía se nutre de la información exhibida por los medios, estos son los que contribuyen con la producción de los marcos referenciales que conducen a la población a generar un cierto sentido y un saber, respecto a lo que sucede en el quehacer diario.

La influencia que ejercen los medios se evidencia dependiendo del grado de la credibilidad que estos tienen en la población. Desde esta perspectiva, los medios de comunicación, como gestores de la información, son percibidos por sus públicos como instituciones "confiables" y en algunos casos "no confiables", a la hora de difundir una noticia, esto depende de la óptica, de las percepciones, y de los sesgos. Indudablemente, a través de estos, la ciudadanía refuerza la credibilidad, por lo que urge trabajar en la calidad y el tratamiento que el medio le da a la información que generan, reproducen y transmiten.

En este sentido el tratamiento de la información se constituye en uno de los elementos preponderantes para la imagen y respeto que deben tener los medios de comunicación, tiene una relación directa con el tratamiento que estos les brinden a la información. Umberto Eco expone algunos criterios respecto a ciertas problemáticas como por ejemplo el valor ético; honestidad e imparcialidad, el riesgo de las redes por la inmediatez, la contratación de fuentes, la objetividad, son retos que los medios de comunicación enfrentan en el tratamiento de la información.

Barthes Roland publicó, un ensayo denominado: "El grado cero de la escritura", en este se plantea la posibilidad de una existencia de una escritura neutra, es decir sin prejuicio, obcecación, o percepciones un texto limpio de sesgo. Es decir, en el desarrollo de su escrito, el autor plantea un debate utópico donde los escritores, por el hecho de ser humanos están sujetos a sus experiencias, emociones y doctrinas que marcan su línea ideológica al momento de redactar (Barthes, 2011).

\section{Desarrollo}

El desarrollo de la tecnología con los diferentes instrumentos convencionales y tecnológicos que sirven para posibilitar un mensaje, han contribuido para que en la actualidad se pueda utilizar de una diversa gama de medios tecnológicos, es decir, que, a través de estos, se mantiene en constante información a la comunidad, anunciando y denunciando los acontecimientos ocurridos en el día a día, y en algunos casos han contribuidos con la solución de alguna problemática social.

Los medios de comunicación locales como nacionales se fueron aliando con el poder económico y político, formando los monopolios y oligopolios de la información; por este accionar el periodista Antonio Espina le denomina como el "cuarto poder" (Espina, 2010). De esta forma que fueron perdido la naturaleza inherente a su función y existencia, esta alianza provoco en estos, una mirada en ocasiones distorsionada de la realidad en otras los medios fueron construyendo la verdad, con el fin de mantener información hegemónica, que se ha operacionalizado a través del acto comunicativo, sistema de educación, sistemas religiosos, sistemas políticos, 


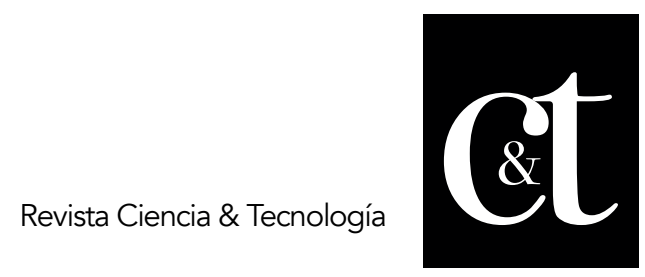

No. 19, 31 de julio de 2018

ISSN impreso: 1390 - 6321

entre otros, que han sometido a la población sistemáticamente a través de los medios de comunicación, hasta neutralizar cualquier tipo de accionar.

Sin embargo, para poder analizar el accionar de los medios de comunicación, se debe analizar cuál es el contenido del producto que estos ofrecen, porque es en la estructuración y socialización de este producto donde se encuentra la verdadera manifestación del control del poder sobre las mentes, este mensaje puede calar hondo. En el siglo XXI el peligro que se cierne sobre algunos países de América Latina es que los medios de comunicación públicos se conviertan en verdaderos aparatos ideológicos de gobierno, tal como se convirtieron algunos medios de comunicación privados, para disfrutar del poder, moverse en él y vivir de él.

Ahora bien, en este sentido se tiene que hacer una distinción entre los medios públicos, privados, y comunitarios, cada uno tiene una función y su campo de acción determinada por la Ley. Esta regulación orienta su accionar respecto al servicio que oferta a la comunidad. En todo caso La Constitución de la república del 2008 presenta en la sección tercera con referencia a lo que es la Comunicación e Información, con respecto al Artículo. 16.- Todas las personas, en forma individual - colectiva, tienen derecho a una comunicación libre, intercultural, incluyente, diversa y participativa, en todos los ámbitos de la interacción social, por cualquier medio y forma, en su propia lengua y con sus propios símbolos. La comunicación se constituye en misma como un derecho, del que todo ciudadano se debe agradar.

Art. 18.- Todas las personas, en forma individual o colectiva, tienen derecho a buscar, recibir, intercambiar, producir y difundir información veraz, verificada, oportuna, contextualizada, plural, sin censura previa acerca de los hechos, acontecimientos y procesos de interés general, y con responsabilidad ulterior (Asamblea Nacional del Ecuador, 2008: 26).

A través de la ley, el Estado garantiza el fortalecimiento y la creación de estos medios, pero lo más importante es que estos estén al servicio de la ciudadanía, especialmente para los territorios en los que se carezca o se tenga limitación en el campo de la comunicación, en este sentido vale plantearse la interrogante, cuál debe ser la función que tiene un medio de comunicación y a quién responde en su accionar. Al gobierno de turno, a los poderes fácticos o a la ciudadanía.

Como consecuencia de lo ante expuesto, es interesante analizar hasta qué punto los medios ejercen un control sobre los pensamientos, la conducta y voluntad, para que consciente o inconscientemente se tome una decisión, determinar los hábitos, configurar las costumbres y determinar el cómo se asume la vida, Chomsky en su libro: "Ilusiones necesarias", afirma: "(...) el control del pensamiento, tal y como se lleva a cabo por medio de la acción de los medios de comunicación nacionales y elementos afines de la cultural intelectual de elite" (Chomsky, 1992), es decir, que todo mensaje emitido que se deriva la información y sus prácticas discursivas en los medios de comunicación, tienen un fin, esto es mantener la hegemonía de ciertos grupos de elites económicos, políticos.

Los distintos medios de comunicación luchan por la supremacía de esta hegemonía de tal forma que se observa a diario en el país es una guerra mediata de información, en el que, el único perdedor es la población. En el siglo XXI, con el desarrollo en la tecnología, se tiene que ser cauto con lo que se observa o lee, debido 


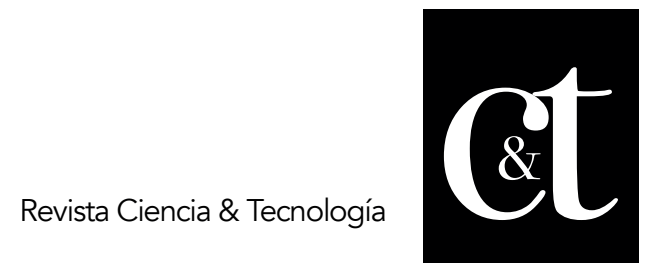

No. 19, 31 de julio de 2018

ISSN impreso: 1390 - 6321

a que se puede actuar sin conciencia moral y esto lo puede llevar a los medios a construir una verdad. Noah Chomsky manifiesta que "un Estado totalitario puede estar satisfecho con niveles inferiores de lealtad hacia las verdades requeridas. Es suficiente que la gente obedezca. Pero en un orden político democrático, siempre se corre el peligro de que el pensamiento independiente se pueda traducir en la acción política, de manera que es importante eliminar la amenaza de raíz (Chomsky, 1999). Por estos motivos es que el gobierno debe asumir un rol preponderante en la vida del país evitando de ser posible el amordazamiento, penalización y apresamiento de los libres pensadores que con cláusula conciencia opinen, diriman, denuncien, todas las situaciones que puedan afectar el desarrollo pleno de la nación.

\section{La construcción de Ciudadanía}

El término construcción de ciudadanía conceptualmente para muchos en la actualidad es nuevo, pero no operativamente, ya que de una u otra forma de alguna manera se ha ido fortaleciendo con nuestro trabajo empeño y empuje la institucionalidad del estado, sin embargo, el nuevo marco constitucional nos invita a reflexionar más profundamente en este tema que es definitivamente muy importante para el ejercicio plano de la responsabilidad como ciudadano del Ecuador.

Carpizo (1999) explica que los medios de comunicación masiva contribuyen en gran parte a fijar las maneras de pensamiento de la sociedad; a establecer la agenda de los asuntos políticos, sociales y económicos que se discuten; a crear o a destruir la reputación de una organización, persona o grupo de personas; proporcionan información y elementos para que la persona o el público construyan, ponderen y formen sus opiniones. Son en muchas ocasiones los intermediarios entre la sociedad y el poder político. Son un poder porque poseen los instrumentos y los mecanismos que les dan la posibilidad de imponerse; que condicionan o pueden condicionar la conducta de otros poderes, organizaciones 0 individuos con independencia de su voluntad y de su resistencia. De modo que es impresionante la capacidad que estos tienen a la hora de interactuar con la población.

Ahora bien, el término ciudadanía es aplicado y fundamentado en la Constitución de la República de 2008, en el artículo 30 sección sexta, en hábitat y vivienda, "Las personas tienen derecho al disfrute pleno de la ciudad, (...), en el ejercicio pleno de la ciudadanía". La pregunta es cómo se va a ejercer este disfrute pleno, si se desconoce por parte de la ciudadanía los derechos que les asiste, entonces aquí tenemos una gran dificultad, ya que los medios no se están involucrando en construir ciudadanía, partiendo desde lo más puntual que es marco constitucional, que se transforma en el referente ya que ahí se tiene los lineamientos que la sociedad ecuatoriana debe asumir y desde ahí articular todos los aspectos de la vida el ser humano en sociedad. La Constitución expresa en el Artículo 95 lo siguiente: Art. 95.- Las ciudadanas y ciudadanos, en forma individual y colectiva, participarán de manera protagónica en la toma de decisiones, planificación y gestión de los asuntos públicos, y en el control popular de las instituciones del Estado y la sociedad, y de sus representantes, en un proceso permanente de construcción del poder ciudadano. La participación se orientará por los principios de igualdad, autonomía, deliberación pública, respeto a la diferencia, control popular, solidaridad e interculturalidad. La participación de la ciudadanía en todos los asuntos de interés público es un derecho, que se ejercerá a través de los 


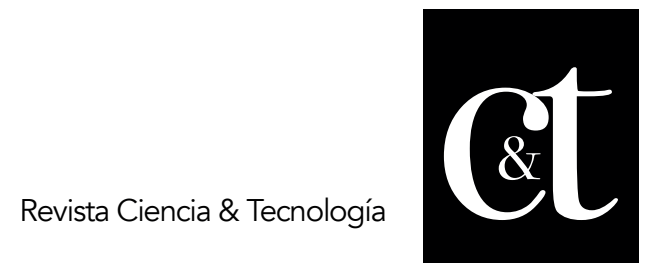

No. 19, 31 de julio de 2018

ISSN impreso: 1390 - 6321

mecanismos de la democracia representativa, directa y comunitaria.

La comunicación es el único medio, o el medio principal para que los ciudadanos puedan intercambiar información, ideas o pensamientos, y llevar una interacción social en el medio, así como consta en el siguiente artículo: "El sistema de comunicación social asegurará el ejercicio de los derechos de la comunicación, la información y la libertad de expresión, y fortalecerá la participación ciudadana (Art. 384: 137). Ahora bien, como construir ciudadanía y evitar el discurso político es uno de los lineamientos que se deben fortalecer en este proceso de construcción.

El Estado a través de los medios de comunicación debe fomentar espacios de intercambios reales de participación con la ciudadanía, además de fomentar el paradigma del dialogo, establecer relaciones de gobernanza a través de una verdadera democracia participativa, esta fase e conciencia se manifiesta, cuando existe una verdadera construcción de ciudadanía, es casi imposible pasar al nivel de participación sin el entendimiento de lo que significa la ciudadanía.

Dentro del marco del foro que se llevó a cabo sobre el cumplimiento y resultados de la aplicación de la ley de acceso a la información en Ecuador, el Ec. Boris Cornejo ${ }^{3}$ señaló que [...] "...una sociedad no puede ser definida como una sociedad democrática, abierta, que estimula la participación, que vela por los derechos de la gente si no garantiza al mismo tiempo que los ciudadanos conozcan qué es lo que pasa, cómo se toman las decisiones, cómo se apropian y se usan los recursos, cómo se rinde cuentas, en definitiva, sobre el devenir de los hechos sociales, económicos, políticos, culturales que suceden en su país (Yandún, 2009)" ${ }^{4}$.

El acceso a la información garantiza a la ciudadanía que se están transparentando el accionar del representante gubernamental. Porque al ser dicha información pública, es de pertenencia de la ciudadanía, tal como lo señala la Comisión Interamericana de Derechos Humanos, asumir este proceso como acto de respeto a la ciudadanía, también es construir ciudadanía...

Por lo tanto, el recurso informacional es de vital trascendencia, para que la ciudadanía pueda ejercer su derecho a mantenerse informado y poder ejercer el derecho de la toma de decisiones, que es vital para la transformación social, puesto que provoca un impacto en las relaciones entre los individuos, reduciendo la incertidumbre de lo que acontece. Pero dichos recursos, en algunas ocasiones no son fáciles, por la falta de medios, económicos y tecnológicos, de parte del ente gubernamental; además sin dejar de señalar la falta de cultura en este tema, lo que impide el acceso a la información, directa y plena. Pero ¿Qué es la información? ¿Cuál es su verdadera trascendencia? Coutin y Ponjuan, señalados en el documento, indican que la información es: [...] "...como un conjunto de datos ordenados de manera significativa, que relacionan la manera como suceden las cosas, hechos, fenómenos y cómo se vinculan a su vez con otros; permitiendo conocer situaciones, resolver problemas y satisfacer las necesidades" (Fernández, Belloso y Delgado, 2008).

\section{Método}

En la investigación se utilizó el enfoque culi-cuantitativo, se analiza la problemática dentro de las delimitaciones planteadas para estudiar el fenómeno dentro de las 
particularidades, medios de comunicación, construcción de ciudadanía, como caso de estudio los medios de la provincia de Santa Elena.

El tipo de investigación, a nivel territorial es exploratoria con algunas particularidades descriptivas, lo que permitirá conocer el comportamiento de las variables relacionadas con la investigación, los instrumentos para la recolección de datos utilizados son; el análisis documental, la encuesta, y la entrevista.

Para determinar el universo se los medios de comunicación domiciliados en la provincia de Santa Elena. Según el consejo de Regulación y Desarrollo de la Información y Comunicación (CORDICOM), los medios de comunicación que operan en la provincia son: 15 radios; de los cuales 8 tiene en su franja informativos, 6 revistas, 10 periódicos y 2 canales de televisión (CORDICOM, 2015), que de una u otra forma han colaborado para mantener a la provincia informada de lo que acontece a nivel local, regional y nacional. Sin embargo, poco han hecho en relación con el trabajo con la comunidad.

Santa Elena, es una de las 24 provincias de la República del Ecuador en las coordenadas $2^{\circ} 13^{\prime} 36^{\prime \prime} \mathrm{S} 80^{\circ} 51^{\prime} 30^{\prime \prime} \mathrm{O}$, con una población de 308.963 habitantes, distribuida en los tres cantones. A nivel provincial, los hombres representan el $50,8 \%$ de la población frente a un $49,2 \%$ de mujeres. De acuerdo con los datos del Censo de Población y Vivienda realizado en el 2010 (INEC, 2010). Además, registra una población flotante de más de 200,000 personas en temporada alta, los meses de enero a mayo.

Para la recolección de los datos se aplicó una encuesta a una muestra de 399 personas, calculada a partir de la población empadronada (209.477 habitantes).

\footnotetext{
3 Boris Cornejo es Economista, Máster en Filosofía del Desarrollo por la Universidad de Cambridge, Inglaterra. Vicepresidente y fundador de la Fundación Esquel.

4 Véase Memorias del Foro Transparencia y Acceso a la Información Pública del Ecuador, CEDA, ECUADOR, 2009, INTERNET, http://www.ceda.org.ec/
} 


\section{Resultados}

Tabla 1. Medios de Comunicación Santa Elena

\begin{tabular}{|c|c|c|}
\hline Medios & $\mathbf{n}$ & $\%$ \\
\hline Televisión & 2 & 6 \\
\hline Radio & 14 & 44 \\
\hline Periódicos & 10 & 31 \\
\hline Revista & 6 & 19 \\
\hline Total & 32 & 100 \\
\hline
\end{tabular}

Fuente: CORDICOM 2015

De los medios de comunicación existentes en la provincia, los que mantienen mayor sintonía, son los medios radiales. El análisis de contenido realizado a dichos medios se evidencia en la parrilla la ausencia de programación que contribuya a la construcción de ciudadanía. Con lo que sí han contribuido con la población, es en la emisión de comunicados, servicio social, denuncias puntuales, entre otras situaciones, esto no significa que exista una construcción una planificación en su programación acorde a las necesidades que contribuya a la construcción de ciudadanía.

Tabla 2. Tipo de programación que tienen los medios, segmento radio

\begin{tabular}{|l|c|c|}
\hline Categoria & $\begin{array}{c}\text { Frec. } \\
\text { Absoluta }\end{array}$ & $\%$ \\
\hline Noticias & 220 & $55 \%$ \\
\hline Musical & 125 & $31 \%$ \\
\hline Cultural & 34 & $9 \%$ \\
\hline Deportivo & 20 & $5 \%$ \\
\hline TOTAL & 399 & $\mathbf{1 0 0 \%}$ \\
\hline
\end{tabular}

Fuente: Población provincia de santa Elena 


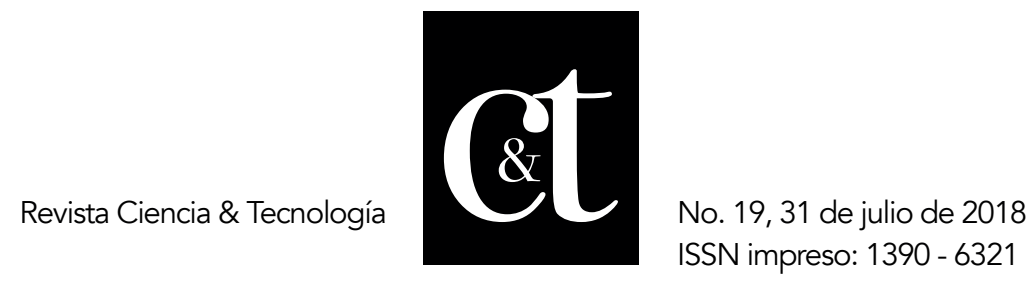

Estos datos indican que los medios de comunicación radiales en la provincia de Santa Elena dedican su parrilla a programas que poco contribuyen a la formación de ciudadanía. Como lo diría Marx, algunos medios de comunicación son el nuevo "opio de los pueblos" (Dognin, 2004). La crítica de la religión desengaña al hombre para que piense, para que actúe y organice su realidad. Un paralelismo. Tienen adormecida la conciencia de los ciudadanos, con la porno-miseria y la info-basura.

Con relación a la información emitida por los medios de comunicación permite evidenciar la realidad, los datos son los siguientes; los encuestados manifiestan que la información emitida por los medios de comunicación no es necesariamente un indicador para evidenciar la realidad, esto puede indicar una problemática escondida, que es la ausencia de una planificación que permita guiar los contenidos, los medios de comunicación se están dejando guiar por la agenda mediática. Los datos de los encuestados se presentan en la siguiente tabla.

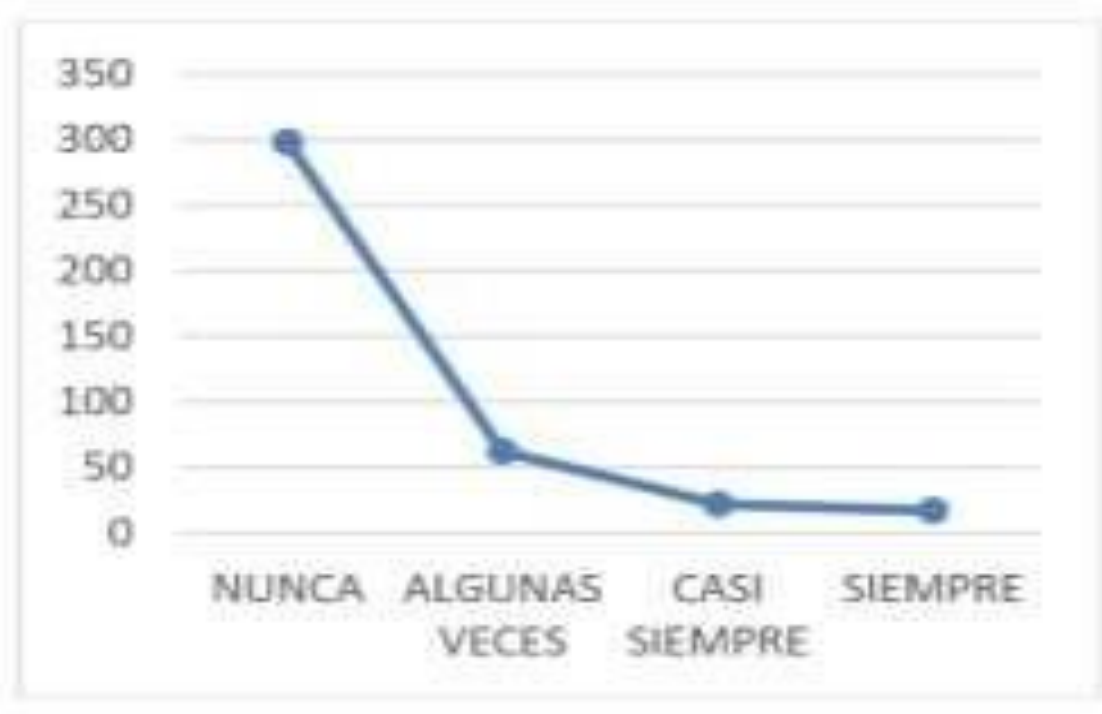

\section{Fuente: Población provincia de santa Elena}

\section{Gráfico 1. Los medios de comunicación evidencian la realidad social}

Como se evidencia en la gráfica con relación a esta pregunta su respuesta va de picada. 


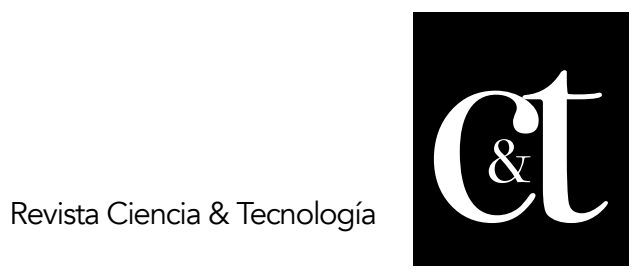

No. 19, 31 de julio de 2018

ISSN impreso: 1390 - 6321

Tabla 3. Relación Pregunta y respuesta

\begin{tabular}{|c|c|c|c|}
\hline OPCIONES & $\begin{array}{c}\text { Frec. } \\
\text { Absoluta }\end{array}$ & $\%$ \\
\hline Denuncias & 200 & $47 \%$ \\
\hline Petitorios & 60 & $15 \%$ \\
\hline Solicitudes & 48 & $11 \%$ \\
\hline Atención & 63 & $14 \%$ \\
\hline Agradecimien. & 28 & $7 \%$ \\
\hline Texto escrito & 28 & $6 \%$ \\
\hline Total & 399 & $100 \%$ \\
\hline
\end{tabular}

\section{Fuente: Población provincia de santa Elena}

En encuesta realizada para medir el grado de participación de los ciudadanos en interacción con los medios de comunicación, según los resultados, respecto a esta pregunta el $47 \%$ de los encuestados indican que interactúan con denuncias realizadas a los medios de comunicación, para que las necesidades sentidas en la comunidad sean resueltas por las autoridades. La respuesta de la autoridad se hace visible inmediatamente.

Según el análisis de las entrevistas que se realizó en la investigación de campo se ha identificado que los medios de comunicación en la actualmente se encuentra en crisis, que el manejo de la información es tratada de acuerdo a la conveniencia del momento, existe alto grado de desconfianza por parte de los habitantes y poca credibilidad en los informes de la noticia, además en el manejo de la información carece de ética profesional, la información es parcializada, y responde a diversos intereses de ciertas personas y grupos de poder. Considera que los medios de comunicación contribuyen con la construcción de ciudadanía.

Los comunicadores expresaron que los medios de comunicación con el hecho de informar a la comunidad se están contribuyendo con la construcción de ciudadanos, que asuman con responsabilidad la ciudadanía. Según esta lógica, los comunicadores al informar a la comunidad están formando ciudadanía responsable y la gente tiene posibilidad de elegir lo que mejor le convenga. Sin embargo, el construir ciudadanía es otro proceso de construcción que debe ser fundamento desde las aulas de clase, familia, instituciones, es decir desde la institucionalización del Estado.

El perfil del personal que labora en los medios de comunicación es importante para construir ciudadanía.

El perfil de los comunicadores en realidad no tiene un patrón determinado, en la provincia de Santa Elena, los comunicadores buscan una oportunidad de 
expresarse, comunicarse, para dar a conocer sus pensamientos, aspiraciones, metas, de colorear una programación con sus ideas y sonrisas, es un gran balcón para satisfacer la necesidad de emitir y receptar ideas para la ciudadanía.

La radio satisface a nichos abandonados durante mucho tiempo que es ese espacio de edad entre los 16 hasta los 35 años, nosotros estamos trabajando bajo esa perspectiva con esa brújula créeme que me llena de mucha satisfacción ver que diariamente son muchos los jóvenes que se identifican con la programación de la radio, con su filosofía y con ese sentir.

Los medios de comunicación deben emplear periodistas con la formación académica. El profesional siempre debe enmarcarse en prepararse académicamente, es importante la preparación en todos los campos iniciando por la preparación ética, un profesional debe estar tomando cursos y preparándose diariamente para conocer los avances en el área comunicativa y así desenvolverse de forma correcta en su labor diaria.

En la actualidad todo fluye tan rápido todos los días nacen nuevos desafíos para quienes tienen la responsabilidad de trabajar en medio de comunicación, hace 20 años en las aulas enseñaban que el lead de la noticia era; el qué, cómo, cuándo, dónde, por qué, hoy todos los ciudadanos tiene la oportunidad de reportar, enviar notas, opinar, sugerir, en otras palabras todos son periodistas, todos tienen un teléfono inteligente para enviar una noticia, esa es una de las virtudes que tiene la web 2.0. El internet se ha convertido en una herramienta fundamental que tiene que ir a la par de la formación académica del comunicador, actualmente más que un comunicador se debe ser ciudadano, capaces de educar a la comunidad. El comunicador tiene que trabajar mucho bajo la perspectiva del servicio a la comunidad, con conciencia social, construir ciudadanía. El construir ciudadanía es un imperativo ético.

Los medios de comunicación en la provincia de Santa Elena son la radio, televisión, revistas y periódicos, entre los periódicos el que se destaca por su aceptación en la comunidad es el diario Súper.

Entre los medios de comunicación de manera general el que tiene mayor aceptación son los radiales, esto se debe a que la radio tiene mayor cobertura rompe con la dinámica del tiempo y la distancia, por lo tanto, es el que los habitantes elijen por ser el medio más rápido a la hora de emitir un mensaje.

Existen algunas características positivas de los medios de comunicación en Santa Elena, como por ejemplo posibilitan que los contenidos de la programación lleguen a lugares muy apartados de la provincia en forma inmediata, en este sentido hace posible que muchas relaciones personales se mantengan unidas 0 , por lo menos, informados.

Estos han contribuido en la comunidad con espacios de información de lo que sucede en la comunidad, sirviendo como un puente entre los diferentes estamentos de la sociedad. La comunicación es el único medio, o el medio principal para que los ciudadanos puedan intercambiar información, ideas o pensamientos, y llevar una interacción social 
El problema básico de los medios es la politización que estos han tenido históricamente ya que han desarrollado su actividad aliados del poder, para mantener la ideología de interés propio o de grupos específicos, para este fin ha limitado su ámbito de acción a entretener, e informar, respecto a temas, entre otros, colaborando muy poco con el desarrollo de pensamiento crítico generador de opinión en la ciudadanía, peor aún construir junto a los actores locales espacios que permitan asumir esa postura crítica.

Otro factor está en el ámbito económico, quien posee el uso de la frecuencia de un medio ha generado de una u otra forma riqueza ya que la comunicación se la ha vendido como un producto es decir, puede generar su propia demanda, visto desde el ámbito empresarial, a estas personas no les interesa el bienestar común, para ellos es el negocio de la comunicación, comercializan las cuñas, realizan pautajes, entre otros negocios, al hacer posible el marketing y anuncios para la comunidad, forjando el mercado de la comunicación.

Por otro lado, en un número considerable de los medios de comunicación de la provincia laboran personas sin título de comunicadores sociales o periodistas ya que resulta en mano de obra más barata el tener que pagarle a una persona no preparada que a un profesional, causando en algunos casos situaciones que van en detrimento del buen convivir ciudadano.

Otro aspecto también importante que se merece la atención es el menoscabo que ha sufrido el profesional de la comunicación precisamente por malos elementos que han mostrado una conducta antiprofesional y antiética, causando también daño a esta noble profesión con mucho acierto el presidente de la República, Economista Rafael Correa los denomino como "sicarios de tinta", indudablemente un pensamiento analógico que cobra vida cuando no se utiliza muy bien este encargo social.

Los medios de comunicación en la actualidad están dejando de lado la ética periodística y se están alineando a determinado proyecto políticos y económicos, no tienen una línea coherente de pensamiento y actitud, esto no puede continuar, ya que los sistemas comunicacionales no se consideran aislados, sino que tienen relación con aspectos de la sociedad y afectan a su forma, de tal forma que es de fundamental importancia replantear su quehacer.

Por ejemplo, la noticia por sus características propias es de interés para la opinión pública, la información errónea que transmita el emisor por falta de investigación o cualquier otro aspecto dejando de lado la veracidad, causará confusión. El público en ocasiones recibe tal cantidad de información que no es capaz de distinguir entre lo errado y lo verdadero, siendo vital que el flujo informativo se desarrolle de manera imparcial con el propósito de que la información sea asimilada de forma adecuada por los receptores, entonces La noticia constituye actualmente una herramienta básica del ser humano en su desarrollo social, ya que le permite mantenerse en contacto con lo que ocurre a su alrededor, la información es un medio de persuasión, llegándose a la conclusión de que la noticia es un instrumento de poder, que ingresa a la mente de los receptores formando juicios sobre determinado tema. En esto radica la importancia de que profesionales en la comunicación estén cubriendo estos espacios. 
Para que la sociedad civil se fortalezca sin ser capturada por procesos de captación del aparato de Estado, es preciso que sea capaz de ser suficientemente autónomo para preservar sus ideas e identidades.

\section{Conclusiones}

Los medios de comunicación poco o nada han contribuido en la construcción de ciudadanía.

Hasta antes de la Ley de Comunicación, no estaba determinado el rol social que tienen los medios de comunicación.

Existe una confusión en cuanto a la que es la información, opinión, expresión, desde la perspectiva de la ciudadanía, del profesional, y del trabajador empírico del medio.

Existe en los medios de comunicación un gran número de personas empíricas en el campo de la comunicación afectando gravemente a los profesionales y a la población por las falencias del acto comunicativo,

La carencia de un código deontológico que permita asumir con responsabilidad el encargo social de la comunicación.

La comunicación para los "dueños de los medios" de las frecuencias concesionadas ha sido el gran negocio. Que con la nueva Ley se termina la fiesta.

\section{Referencias bibliográficas}

Asamblea Nacional del Ecuador (2008). La Constitución de la República del Ecuador. Montecristi.

Barthes, R. (2011). El grado cero de la escritura. Buenos Aires: Siglo XXI.

Carpizo, J. (1999). Los medios de comunicación masiva y el Estado de derecho, la democracia, la política y la ética. Boletín Mexicano de Derecho Compartido, 32(96). Antología sobre teoría y métodos de Comunicación Educativa I.

Chomsky, N. (1999). Control del pensamiento en las sociedades democráticas. En N. Chomsky, Control del pensamiento en las sociedades democráticas (pág. 64). Madrid: Libertarias.

Chomsky, N. (1992). Control del pensamiento en las sociedades democráticas. Libertarias, Madrid. Madrid: Libertarias, Madrid.

CORDICOM (15 de junio de 2015). cordicom.gob.ec/. Obtenido de cordicom.gob.ec/: www.cordicom.gob.ec/wpcontent/.../listado_medios_registrados_15_06_2015.p df

Dognin, P. D. (2004). Introducción a Karl Marx. En P. D. Dognin, Introducción a Karl Marx (págs. 85 -85). Caracas: Universidad Católica Andrés Bello.

Espina, A. (2010). El cuarto poder: cien años de periodismo español. Universidad Estatal de Pensilvania.

Fernández, N., Belloso, N. y Delgado, F. (2008). Recursos informacionales, desarrollo endógeno y participación ciudadana en la Gestión Pública Local. 


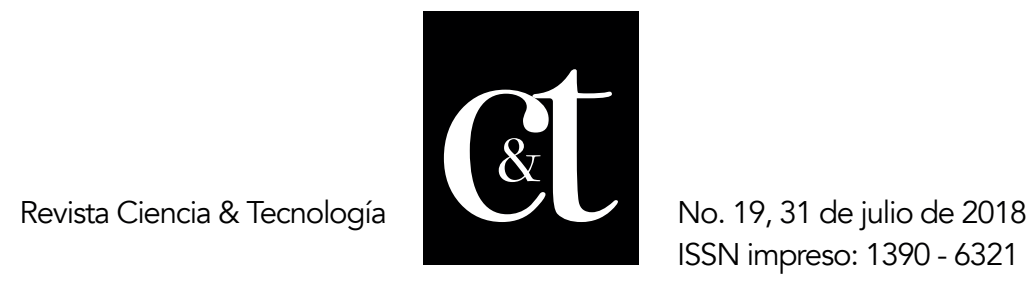

Revista Venezolana de Gerencia, v.13 n.43 Maracaibo sep. 2008.

INEC (2010). Censo de Población y Vivienda. Obtenido de INEC: http//www.inec.gov.ec.base/de/datos.

Yandún, R. V. (2009). Transparencia y acceso a la información pública en el Ecuador. CEDA Centro Ecuatoriano de Desarrollo Ambiental. Quito: CEDA Centro Ecuatoriano de Desarrollo Ambiental. Obtenido de http://www.ceda.org.ec/descargas/publicaciones/MemoriaForoLOTAIP.pdf 